\title{
Oxalic acid as an efficient, cheap, and reusable catalyst for the preparation of quinoxalines via condensation of 1,2-diamines with $\alpha$-diketones at room temperature
}

\author{
Alireza Hasaninejad,*a Abdolkarim Zare, ${ }^{* b}$ Mohammad Reza Mohammadizadeh, ${ }^{a}$ and \\ Mohsen Shekouhy ${ }^{\mathrm{a}}$ \\ ${ }^{a}$ Department of Chemistry, Faculty of Sciences, Persian Gulf University, Bushehr 75169, Iran \\ ${ }^{b}$ Department of Chemistry, Payame Noor University of Bushehr, Bushehr 1698, Iran \\ E-mail:ahassaninejad@yahoo.com,abdolkarimzare@yahoo.com
}

\begin{abstract}
A simple, highly efficient and green procedure for the condensation of aryl and alkyl 1,2diamines with $\alpha$-diketones in the presence of catalytic amount of oxalic acid at room temperature is described. Using this method, quinoxaline derivatives as biologically interesting compounds are produced in high to excellent yields and short reaction times.
\end{abstract}

Keywords: Oxalic acid, quinoxaline, 1,2-diamine, $\alpha$-diketone, green chemistry

\section{Introduction}

The quinoxaline skeleton is a building block for the preparation of substances with pronounced biological activities, such as antimycobacterial, ${ }^{1}$ antidepressant, ${ }^{2}$ and antitumor drugs. ${ }^{3}$ Moreover, quinoxaline ring is a part of various antibiotics, such as Echinomycin, Levomycin and Actinoleutin that are known to inhibit growth of gram positive bacteria ${ }^{4}$ and are active against various transplantable tumors. ${ }^{5}$ They have been also used as building blocks for the synthesis of organic semiconductors. ${ }^{6}$ Quinoxaline derivatives have been also applied for metal cations extraction. $^{7}$ A number of methods have been developed for the synthesis of substituted quinoxalines. ${ }^{8-13}$ The most common method for their preparation relies on the condensation of an aryl 1,2-diamine with a 1,2-dicarbonyl compound. ${ }^{8}$. Furthermore, there are several synthetic routes toward quinoxalines, including Bi-catalyzed oxidative coupling of epoxides with ene-1,2diamines, ${ }^{9}$ heteroannulation of nitroketene $N, S$-aryliminoacetals with $\mathrm{POCl}_{3},{ }^{10}$ cyclization of $\alpha$ arylimino oximes of $\alpha$-dicarbonyl compounds, ${ }^{11}$ and from $\alpha$-hydroxy ketones via a tandem oxidation process using $\mathrm{Pd}(\mathrm{OAc})_{2}$ or $\mathrm{RuCl}_{2}-\left(\mathrm{PPh}_{3}\right)_{3}-\mathrm{TEMPO}^{12}$ as well as $\mathrm{MnO}_{2} \cdot{ }^{13}$ Nevertheless, most of these methods suffer one or more of the following drawbacks: unsatisfactory yield, long reaction time, critical product isolation procedures, the use of expensive and detrimental metal 
precursors, harsh reaction conditions, and no agreement with the green chemistry protocols, which limit their use. As part of our ongoing program to develop more efficient and environmentally benign methods for organic transformations using economic and ecofriendly materials as catalysts and reagents, ${ }^{14}$ we have looked into the synthesis of quinoxaline derivatives via the condensation of 1,2-diamines with $\alpha$-diketones in the presence of catalytic amounts of oxalic acid at room temperature (Scheme 1). It is worth noting that this present method has not the above mentioned drawbacks.

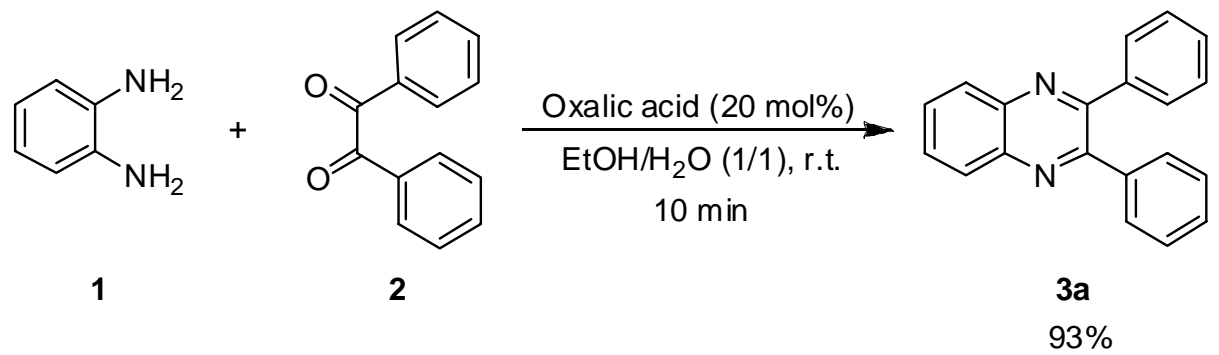

Scheme 1. Condensation of benzene-1,2-diamine with benzil.

\section{Results and Discussion}

In order to find a suitable catalyst for the synthesis of quinoxalines from 1,2-diamines and $\alpha$ diketones, the condensation of benzene-1,2-diamine with benzil was chosen as a model to provide compound 3a (Scheme 1), and its behavior was studied in the presence of various catalysts in EtOH/ $\mathrm{H}_{2} \mathrm{O}$ at room temperature. The results are displayed in Table 1. As it can be seen from Table 1, oxalic acid as an organic catalyst afforded the better results with respect to the inorganic catalysts.

Table 1. The condensation of benzene-1,2-diamine $(1 \mathrm{mmol})$ with benzil $(1 \mathrm{mmol})$ in the presence of different catalysts $(0.2 \mathrm{mmol}, 20 \mathrm{~mol} \%)$ in $\mathrm{EtOH} / \mathrm{H}_{2} \mathrm{O}(1 / 1)$ at room temperature

\begin{tabular}{llll}
\hline Entry & Catalyst & Time $(\mathrm{min})$ & Yield $^{\mathrm{a}}(\%)$ \\
\hline $1^{\mathrm{b}}$ & - & 600 & - \\
2 & Oxalic acid & 10 & 93 \\
3 & $\mathrm{ZnCl}_{2}$ & 240 & 70 \\
4 & $\left.\mathrm{Mn}_{2} \mathrm{OAc}\right)_{2}$ & 240 & 78 \\
5 & $\mathrm{CoCl}_{2}$ & 240 & 81 \\
6 & $\mathrm{Ni}(\mathrm{OAc})_{2}$ & 240 & 68 \\
\hline
\end{tabular}

${ }^{\mathrm{a}}$ Isolated yield. ${ }^{\mathrm{b}}$ Without catalyst. 
To select the best solvent for the reaction, the synthesis of quinoxaline 3a was examined in different solvents (Table 2). As Table 2 indicates, the examined solvents were not efficient separately. Higher yields and shorter reaction times were obtained when the reaction was carried out in $\mathrm{EtOH} / \mathrm{H}_{2} \mathrm{O}(1 / 1)$. Thus, $\mathrm{EtOH} / \mathrm{H}_{2} \mathrm{O}(1 / 1)$ was used as reaction media for all reactions.

Table 2. The effect of various solvents $(20 \mathrm{~mL})$ on the reaction of benzene-1,2-diamine (1 $\mathrm{mmol})$ with Benzil $(1 \mathrm{mmol})$ using oxalic acid $(0.2 \mathrm{mmol})$ at room temperature

\begin{tabular}{llll}
\hline Entry & Solvent & Time (min) & Yield $^{\mathrm{a}}(\%)$ \\
\hline 1 & $\mathrm{EtOH}$ & 60 & 62 \\
2 & $\mathrm{H}_{2} \mathrm{O}$ & 60 & 24 \\
3 & $\mathrm{CHCl}_{3}$ & 60 & 31 \\
4 & $\mathrm{MeCN}$ & 60 & 37 \\
5 & $\mathrm{THF}$ & 60 & 26 \\
7 & $\mathrm{EtOH} / \mathrm{H}_{2} \mathrm{O}(1 / 1)$ & 10 & 93 \\
\hline
\end{tabular}

${ }^{\mathrm{a}}$ Isolated yield

To realize the generality and versatility of the catalyst, different aryl and alkyl 1,2-diamines were condensed with some $\alpha$-diketones. The results are summarized in Table 3. As it is shown in Table 3, when aryl 1,2-diamines were applied, all reactions proceeded efficiently and the desired quinoxalines were obtained in excellent yields and short reaction times. However, alkyl 1,2diamines afforded the corresponding quinoxaline derivatives in slightly lower yields and longer reaction times. In addition, the structure of $\alpha$-diketones did not significantly affect on the results of the reaction.

Table 3. Synthesis of quinoxaline derivatives from 1,2-diamines and $\alpha$-diketones

\begin{tabular}{lllll}
\hline Entry 1,2-Diamine & Time (min) & Yield $^{\mathrm{a}}(\%)$ \\
\hline
\end{tabular}


Table 3. Continued

\begin{tabular}{|c|c|c|c|c|}
\hline Entry & 1,2-Diamine & Product & Time $(\min )$ & Yield $^{\mathrm{a}}(\%)$ \\
\hline 3 & & & 10 & 95 \\
\hline 4 & & & 10 & 92 \\
\hline 5 & & & 60 & 81 \\
\hline 6 & & & 60 & 84 \\
\hline 7 & & & 10 & 92 \\
\hline 8 & & & 10 & 93 \\
\hline 9 & & & 10 & 95 \\
\hline
\end{tabular}


Table 3. Continued

Entry

${ }^{\mathrm{a}}$ Isolated yield.

Ease of recycling of the catalyst is one of the most advantages of our method. For the reaction of benzene-1,2-diamine with benzil no significant loss of the product yield was observed when oxalic acid was used after four times recycling.

\section{Conclusions}

In summary, we have developed an efficient method for the synthesis of quinoxaline derivatives via the condensation of 1,2-diamines with $\alpha$-diketones. This new strategy has several advantages, such as excellent yield, short reaction time, low cost, simple experimental as well as isolation procedures, and finally, it is in agreement with the green chemistry protocols.

\section{Experimental Section}

General Procedures. All chemicals were purchased from Merck or Fluka chemical companies. All known compounds were identified by comparison of their melting points and ${ }^{1} \mathrm{H}$ NMR data with the authentic samples. The ${ }^{1} \mathrm{H}$ NMR $(250 \mathrm{MHz})$ and ${ }^{13} \mathrm{C} \mathrm{NMR}(62.5 \mathrm{MHz})$ were run on a Bruker Avance DPX-250, FT-NMR spectrometer ( $\delta$ in ppm). Mass spectra were recorded on a Shimadzu GC MS-QP 1000 EX apparatus. Microanalyses were performed on a Perkin-Elmer 
240-B microanalyzer. Melting points were recorded on a Büchi B-545 apparatus in open capillary tubes.

Preparation of quinoxalines from 1,2-diamines and $\boldsymbol{\alpha}$-diketones. To a mixture of $\boldsymbol{\alpha}$-diketone $(1 \mathrm{mmol})$, oxalic acid $(0.018 \mathrm{~g}, 0.2 \mathrm{mmol})$ and $\mathrm{EtOH} / \mathrm{H}_{2} \mathrm{O}[1 / 1(\mathrm{v} / \mathrm{v}), 20 \mathrm{~mL}]$ in a $50 \mathrm{~mL}$ roundbottomed flask was added 1,2-diamine $(1 \mathrm{mmol})$, and the resulting mixture was stirred at room temperature for the times reported in Table 3. Then, $\mathrm{H}_{2} \mathrm{O}(20 \mathrm{~mL})$ was added to the reaction mixture, and was allowed to stand at room temperature for $1 \mathrm{~h}$. During this time, crystals of the pure product formed which were collected by filtration and dried. After isolation of the product, the filtrate was extracted with $\mathrm{CHCl}_{3}(2 \times 30 \mathrm{~mL})$. The aqueous layer (including oxalic acid) was separated, and its solvent was evaporated to obtain pure oxalic acid. The recycled catalyst was used for the next run under identical reaction conditions.

2,3-Diphenylquinoxaline (3a). White solid; mp 130-131 ${ }^{\circ} \mathrm{C}$ (Lit. ${ }^{8 \mathrm{a}} \mathrm{mp} 128-129{ }^{\circ} \mathrm{C}$ ); ${ }^{1} \mathrm{H}$ NMR $\left(\mathrm{CDCl}_{3}\right): \delta$ 7.29-7.33 (6H, m), $7.51(4 \mathrm{H}, \mathrm{m}), 7.77(2 \mathrm{H}, \mathrm{m}), 8.21(2 \mathrm{H}, \mathrm{m})$.

6-Methyl-2,3-diphenylquinoxaline (3b). White solid; mp $115-117{ }^{\circ} \mathrm{C}$ (Lit. ${ }^{8 \mathrm{a}} \mathrm{mp} 117-118{ }^{\circ} \mathrm{C}$ ); ${ }^{1} \mathrm{H} \mathrm{NMR}\left(\mathrm{CDC1}_{3}\right): \delta 2.62(3 \mathrm{H}, \mathrm{s}), 7.26-7.34(6 \mathrm{H}, \mathrm{m}), 7.50(4 \mathrm{H}, \mathrm{m}), 7.60(1 \mathrm{H}, \mathrm{d}, J=8.4 \mathrm{~Hz})$, $7.84(1 \mathrm{H}, \mathrm{s}), 8.10(1 \mathrm{H}, \mathrm{d}, J=8.4 \mathrm{~Hz})$.

6,7-Dimethyl-2,3-diphenylquinoxaline (3c). Pale yellow solid; mp 177-179 ${ }^{\circ} \mathrm{C}$ (Lit. ${ }^{8 b} \mathrm{mp} 172$ $\left.{ }^{\circ} \mathrm{C}\right) ;{ }^{1} \mathrm{H} \mathrm{NMR}\left(\mathrm{CDC1}_{3}\right): \delta 2.46(6 \mathrm{H}, \mathrm{s}), 7.19-7.27(6 \mathrm{H}, \mathrm{m}), 7.42(4 \mathrm{H}, \mathrm{d}, J=9.0 \mathrm{~Hz}), 7.58(2 \mathrm{H}, \mathrm{s})$. 2,3-Diphenylbenzo[g]quinoxaline (3d). Yellow solid; mp 186-188 ${ }^{\circ} \mathrm{C}$ (Lit. ${ }^{8 \mathrm{c}} \mathrm{mp} 187-188{ }^{\circ} \mathrm{C}$ ); ${ }^{1} \mathrm{H} \mathrm{NMR}\left(\mathrm{CDC1}_{3}\right): \delta$ 7.30-7.34 (6H, m), 7.48-7.53 (6H, m), 8.03 (2H, m), 8.59 (2H, s).

2,3-Diphenyl-4a,5,6,7,8,8a-hexahydroquinoxaline (3e). Pale yellow solid; mp 168-170 ${ }^{\circ} \mathrm{C}$ (Lit. $\left.{ }^{8 \mathrm{~b}} \mathrm{mp} 167{ }^{\circ} \mathrm{C}\right) ;{ }^{1} \mathrm{H} \mathrm{NMR}\left(\mathrm{CDCl}_{3}\right): \delta 1.36(2 \mathrm{H}, \mathrm{m}), 1.50(2 \mathrm{H}, \mathrm{m}), 1.69(2 \mathrm{H}, \mathrm{m}), 1.95(2 \mathrm{H}, \mathrm{m})$, $2.96(2 \mathrm{H}, \mathrm{m}), 7.29-7.37(6 \mathrm{H}, \mathrm{m}) 7.48(4 \mathrm{H}, \mathrm{m})$.

5,6-Diphenyl-2,3-dihydropyrazine (3f). White solid; mp $160-162{ }^{\circ} \mathrm{C}$ (Lit. ${ }^{8 b} \mathrm{mp} 158{ }^{\circ} \mathrm{C}$ ); ${ }^{1} \mathrm{H}$ $\operatorname{NMR}\left(\mathrm{CDC1}_{3}\right): \delta 3.80(4 \mathrm{H}, \mathrm{s}), 7.26-7.34(6 \mathrm{H}, \mathrm{m}), 7.48(4 \mathrm{H}, \mathrm{m})$.

2,3-Bis(4-methoxyphenyl)quinoxaline (3g). Pale yellow solid; mp $148-150{ }^{\circ} \mathrm{C}$ (Lit. ${ }^{8 \mathrm{~d}} \mathrm{mp} 151$ $\left.152{ }^{\circ} \mathrm{C}\right) ;{ }^{1} \mathrm{H} \mathrm{NMR}\left(\mathrm{CDC1}_{3}\right): \delta 3.84(6 \mathrm{H}, \mathrm{s}), 6.85(4 \mathrm{H}, \mathrm{d}, J=8.6 \mathrm{~Hz}), 7.47(4 \mathrm{H}, \mathrm{d}, J=8.6 \mathrm{~Hz})$, $7.68(2 \mathrm{H}, \mathrm{m}), 8.19(2 \mathrm{H}, \mathrm{m})$.

2,3-Bis(4-methoxyphenyl)-6-methylquinoxaline (3h). Yellow solid; mp 129-131 ${ }^{\circ} \mathrm{C}$ (Lit. ${ }^{8 \mathrm{~d}} \mathrm{mp}$ 125-127 $\left.{ }^{\circ} \mathrm{C}\right) ;{ }^{1} \mathrm{H}$ NMR $\left(\mathrm{CDC1}_{3}\right): \delta 1.58(3 \mathrm{H}, \mathrm{s}), 3.88(6 \mathrm{H}, \mathrm{s}), 6.65(4 \mathrm{H}, \mathrm{d}, J=8.6 \mathrm{~Hz}), 7.05(4 \mathrm{H}$, $\mathrm{d}, J=7.6 \mathrm{~Hz}), 7.61(1 \mathrm{H}, \mathrm{d}, J=8.5 \mathrm{~Hz}), 7.90(1 \mathrm{H}, \mathrm{s}), 8.09(1 \mathrm{H}, \mathrm{d}, J=8.5 \mathrm{~Hz})$.

6-Methyl-2,3-di-p-tolylquinoxaline (3i). Pale yellow solid; mp 138-139 ${ }^{\circ} \mathrm{C}$ (Lit. $\left.{ }^{8 \mathrm{c}} \mathrm{mp} 137{ }^{\circ} \mathrm{C}\right)$; ${ }^{1} \mathrm{H}$ NMR $\left(\mathrm{CDC1}_{3}\right): \delta 2.34(6 \mathrm{H}, \mathrm{s}), 2.51(3 \mathrm{H}, \mathrm{s}), 7.17(4 \mathrm{H}, \mathrm{d}, J=8.0 \mathrm{~Hz}), 7.46(4 \mathrm{H}, \mathrm{d}, J=8.0$ $\mathrm{Hz}), 7.58(1 \mathrm{H}, \mathrm{d}, J=8.5), 7.93(1 \mathrm{H}, \mathrm{s}), 8.03(1 \mathrm{H}, \mathrm{d}, J=8.5 \mathrm{~Hz})$.

2,3-Di-p-tolylbenzo[g]quinoxaline (3j). Yellow solid; mp 196-198 ${ }^{\circ} \mathrm{C}$ (Lit. ${ }^{8 \mathrm{c}} \mathrm{mp} 198{ }^{\circ} \mathrm{C}$ ); ${ }^{1} \mathrm{H}$ NMR $\left(\mathrm{CDC1}_{3}\right): \delta 2.35(6 \mathrm{H}, \mathrm{s}), 7.16(4 \mathrm{H}, \mathrm{d}, J=8.0 \mathrm{~Hz}), 7.45-7.50(6 \mathrm{H}, \mathrm{m}), 8.05(2 \mathrm{H}, \mathrm{m}), 8.62$ $(2 \mathrm{H}, \mathrm{s})$.

2-Methyl-3-phenylquinoxaline (3k). Orange solid; mp 57-59 ${ }^{\circ} \mathrm{C}$ (Lit. ${ }^{8 \mathrm{c}} \mathrm{mp} 56{ }^{\circ} \mathrm{C}$ ); ${ }^{1} \mathrm{H}$ NMR $\left(\mathrm{CDC1}_{3}\right): \delta 2.81(3 \mathrm{H}, \mathrm{s}), 7.45-7.51(3 \mathrm{H}, \mathrm{m}), 7.62-7.71(4 \mathrm{H}, \mathrm{m}), 8.06-8.13(2 \mathrm{H}, \mathrm{m})$. 
9,10-Dimethylacenaphtho[1,2-b]quinoxaline (3I). Yellow solid; mp 304-306 ${ }^{\circ} \mathrm{C}$; ${ }^{1} \mathrm{H}$ NMR $\left(\mathrm{CDC1}_{3}\right): \delta 2.51(6 \mathrm{H}, \mathrm{s}), 7.78(2 \mathrm{H}, \mathrm{m}), 7.89(2 \mathrm{H}, \mathrm{s}), 8.03(2 \mathrm{H}, \mathrm{m}), 8.34(2 \mathrm{H}, \mathrm{m}) ;{ }^{13} \mathrm{C}$ NMR $\left(\mathrm{CDC1}_{3}\right): \delta 20.3,121.5,127.8,128.0,128.6,128.9,129.1,139.5,140.00,148.5,153.3 ; \mathrm{MS} \mathrm{m} / \mathrm{z}$ : $282\left(\mathrm{M}^{+}\right)$; Anal. calcd. for $\mathrm{C}_{20} \mathrm{H}_{14} \mathrm{~N}_{2}$ : C, 85.08; H, 5.00; N, 9.92. Found: C, 85.32; H, 5.16; N, 9.73 .

\section{Acknowledgements}

The authors thank Persian Gulf University and Payame Noor University of Bushehr Research Councils for financial support of this work.

\section{References and Notes}

1. Seitz, L. E.; Suling, W. J.; Reynolds, R. C. J. Med. Chem. 2002, 45, 5604.

2. Badran, M. M.; Botros, S.; El-Gendy, A. A.; Abdou, N. A.; El-Assi, H.; Salem, A. Bull. Pharm. Sci. 2001, 24, 135.

3. Hazeldine, S. T.; Polin, L.; Kushner, J.; Paluch, J.; White, K.; Edelstein, M.; Palomino, E.; Corbett, T. H.; Horwitz, J. P. J. Med. Chem. 2001, 44, 1758.

4. (a) Dell, A.; Williams, D. H.; Morris, H. R.; Smith, G. A.; Feeney, J.; Roberts, G. C. K. J. Am. Chem. Soc. 1975, 97, 2497. (b) Bailly, C.; Echepare, S.; Gago, F.; Waring, M.; J. AntiCancer Drug Des. 1999, 14, 291.

5. Sato, K.; Shiratori, O.; Katagiri, K. J. Antibiot. 1967, 20, 270.

6. (a) Dailey, S.; Feast, J. W.; Peace, R. J.; Sage, I. C.; Till, S.; Wood, E. L. J. Mater. Chem. 2001, 11, 2238. (b) O’Brien, D.; Weaver, M. S.; Lidzey, D. G.; Bradley, D. D. C. Appl. Phys. Lett. 1996, 69, 881 .

7. Bouabdallah, I.; Zidane, I.; Touzani, R.; Hacht, B.; Ramdani, A. Arkivoc 2006, (x), 77.

8. (a) Heravi, M. M.; Bakhtiari, K.; Tehrani, M. H.; Javadi, N. M.; Oskooie, H. A. Arkivoc 2006, (xvi), 16. (b) Darabi, H. R.; Mohandessi, S.; Aghapoor, K.; Mohsenzadeh, F. Catal. Commun. 2007, 389. (c) More, S. V.; Sastry, M. N. V.; Yao, C.-F. Green Chem. $2006,91$. (d) Heravi, M. M.; Taheri, S.; Bakhtiari, K.; Oskooie, H. A. Catal. Commun. 2007, 211. (e) Zhao, Z.; Wisnoski, D. D.; Wolkenberg, S. E.; Leister, W. H.; Wang, Y.; Lindsley, C. W. Tetrahedron Lett. 2004, 45, 4873. (f) Bhosale, R. S.; Sarda, S. R.; Ardhapure, S. S.; Jadhav, W. N.; Bhusare, S. R.; Pawar, R. P.; Tetrahedron Lett. 2005, 46, 7183. (g) More, S. V.; Sastry, M. N. V.; Wang, C.-C.; Yao, C.-F. Tetrahedron Lett. 2005, 46, 6345. (h) Heravi, M. M.; Bakhtiari, K.; Bamoharram, F. F.; Tehrani, M. H. Monatsh. Chem. 2007, 138, 465. (i) Oskooie, H. A.; Heravi, M. M.; Bakhtiari, K.; Taheri, S. Monatsh. Chem. 2007, 138, 875.

9. Antoniotti, S.; Duńach, E. Tetrahedron Lett. 2002, 43, 3971.

10. Venkatesh, C.; Singh, B.; Mahata, P. K.; Ila, H.; Junjappa, H. Org. Lett. 2005, 7, 2169. 
11. Xekoukoulotakis, N. P.; Hadjiantoniu-Maroulis, C. P.; Maroulis, A. J. Tetrahedron Lett. 2000, 41, 10299.

12. Robinson, R. S.; Taylor, R. J. K. Synlett 2005, 1003.

13. (a) Raw, S. A.; Wilfred, C. D.; Taylor, R. J. K. Org. Biomol. Chem. 2004, 788. (b) Raw, S. A.; Wilfred, C. D.; Taylor, R. J. K. Chem. Commun. 2003, 2286.

14. (a) Hasaninejad, A.; Zare, A.; Sharghi, H.; Shekouhy, M. Arkivoc 2008, (xi), 64. (b) Zare, A.; Hasaninejad, A.; Khalafi-Nezhad, A.; Parhami, A.; Moosavi Zare, A. R. J. Iran. Chem. Soc. 2008, 5, 100. (c) Zare, A.; Hasaninejad, A.; Beyzavi, M. H.; Parhami, A.; Moosavi Zare, A. R.; Khalafi-Nezhad, A.; Sharghi, H. Can. J. Chem. 2008, 86, 317. (d) Hasaninejad, A.; Parhami, A.; Zare, A.; Khalafi-Nezhad, A.; Nasrolahi Shirazi, A.; Moosavi Zare, A. R. Polish J. Chem. 2008, 82, 565. (e) Zare, A.; Hasaninejad, A.; Khalafi-Nezhad, A.; Moosavi Zare, A. R.; Parhami, A.; Nejabat, G. R. Arkivoc 2007, (i), 58. (f) Zare, A.; Hasaninejad, A.; Khalafi-Nezhad, A.; Moosavi Zare, A. R.; Parhami, A. Arkivoc 2007, (xiii), 105. (g) Hasaninejad, A.; Zare, A.; Sharghi, H.; Niknam, K.; Shekouhy, M. Arkivoc 2007, (xiv), 39. (h) Imanzadeh, G. H.; Zare, A.; Khalafi-Nezhad, A.; Hasaninejad, A.; Moosavi Zare, A. R.; Parhami, A. J. Iran. Chem. Soc. 2007, 4, 467. (i) Khalafi-Nezhad, A.; Zare, A.; Parhami, A.; Soltani Rad, M. N.; Nejabat, G. R. J. Iran. Chem. Soc. 2007, 4, 271. (j) Zare, A.; Hasaninejad, A.; Moosavi Zare, A. R.; Parhami, A.; Sharghi, H.; Khalafi-Nezhad, A. Can. J. Chem. 2007, 85, 438. (k) Hasaninejad, A.; Zare, A.; Sharghi, H.; Shekouhy, M.; Khalifeh, R.; Salimi Beni, A.; Moosavi Zare, A. R. Can. J. Chem. 2007, 85, 416. (1) Khalafi-Nezhad, A.; Parhami, A.; Soltani Rad, M. N.; Zolfigol, M. A.; Zare, A. Tetrahedron Lett. 2007, 48, 5219. (m) Hasaninejad, A.; Sharghi, H. Phosphorus, Sulfur, and Silicon 2007, 182, 873. (n) Imanzadeh, G. H.; Khalafi-Nezhad, A.; Zare, A.; Hasaninejad, A.; Moosavi Zare, A. R.; Parhami, A. J. Iran. Chem. Soc. 2007, 4, 229. (o) Khalafi-Nezhad, A.; Zare, A.; Parhami, A.; Soltani Rad, M. N.; Nejabat, G. R. Phosphorus, Sulfur, and Silicon 2007, 182, 657. (p) Hasaninejad, A.; Zare, A. J. Sulfur Chem. 2007, 28, 357. (q) Khalafi-Nezhad, A.; Zare, A.; Parhami, A.; Soltani Rad, M. N.; Nejabat, G. R. Synth. Commun. 2006, 36, 3549. 